\title{
Total Internal Reflection Fluorescence Microscopy in Cell Biology
}

\author{
Daniel Axelrod \\ Department of Physics \& Biophysics Research Division, \\ University of Michigan, Ann Arbor, MI 48109, USA, \\ daxelrod@umich.edu
}

Key events in cellular trafficking occur at the cell surface, and it is desirable to visualize these events without interference from other regions deeper within. This review describes a microscopy technique based on total internal reflection fluorescence which is well suited for optical sectioning at cell-substrate regions with an unusually thin region of fluorescence excitation. The technique has many other applications as well, most notably for studying biochemical kinetics and single biomolecule dynamics at surfaces. A brief summary of these applications is provided, followed by presentations of the physical basis for the technique and the various ways to implement total internal reflection fluorescence in a standard fluorescence microscope.

Key words: cell-substrate contact, evanescent, membrane, secretion, surface, optical section

Received 30 July 2001, revised and accepted for publication 20 August 2001

Total internal reflection fluorescence (TIRF) microscopy (also called 'evanescent wave microscopy') provides a means to selectively excite fluorophores in an aqueous or cellular environment very near a solid surface (within $\leq 100 \mathrm{~nm}$ ) without exciting fluorescence from regions farther from the surface (1). Fluorescence excitation by this thin zone of electromagnetic energy (called an 'evanescent field') results in images with very low background fluorescence, virtually no out-offocus fluorescence, and minimal exposure of cells to light at any other planes in the sample. Figure 1 shows an example of TIRF on intact living cells in culture, compared with standard epi-fluorescence. The unique features of TIRF have enabled numerous applications in biochemistry and cell biology, as follows.

(a) Selective visualization of cell/substrate contact regions. TIRF can be used qualitatively to observe the position, extent, composition, and motion of contact regions, even in samples in which fluorescence elsewhere would otherwise obscure the fluorescent pattern (2). A variation of TIRF to identify cell-substrate contacts involves doping the solution surrounding the cells with a nonadsorbing and nonpermeable fluorescent volume marker; focal contacts then appear relatively dark $(3,4)$. Although TIRF cannot view deeply into thick cells, it can display with high contrast the fluorescencemarked submembrane filament structure at the substrate contact regions (5).

(b) Visualization and spectroscopy of single molecule fluorescence near a surface (6-12). The purpose here is to observe the properties of individual molecules without the ensemble averaging inherent in standard spectroscopies on bulk materials, thereby enabling detection of kinetic features and states that otherwise are obscured. Related to single molecule detection is the capability of seeing fluorescence fluctuations as fluorescent molecules enter and leave the thin evanescent field region in the bulk. These fluctuations (which are visually obvious in TIRF) can be quantitatively autocorrelated in a technique called fluorescence correlation spectroscopy (FCS) to obtain kinetic information about the molecular motion (13).

(c) Tracking of secretory granules in intact cells before and during the secretory process. The thin evanescent field allows small intensity changes to be interpreted as small motions of granules in the direction normal to the substrate with precision as small as $2 \mathrm{~nm}$, much smaller than the light microscope resolution limit, and in some cases, dispersal of granule contents interpreted as exocytosis (14-29).

(d) Measurements of the kinetic rates of binding of extracellular and intracellular proteins to cell surface receptors and artificial membranes. Some of these studies combine TIR with fluorescence recovery after photobleaching (FRAP) or FCS (30-42). TIR/FRAP additionally can be used to measure lateral surface diffusion coefficients along with on/off kinetics of reversibly adsorbed fluorescent molecules.

(e) Micromorphological structures and dynamics on living cells. By utilizing the unique polarization properties of the evanescent field of TIR, endocytotic or exocytotic sites, ruffles and other submicroscopic irregularities can be highlighted (43). By combining TIRF with atomic force microscopy, stress-strain relationships can be directly measured on living cells (44).

(f) Long-term fluorescence movies of cells during development in culture. Since the cells are exposed to exci- 

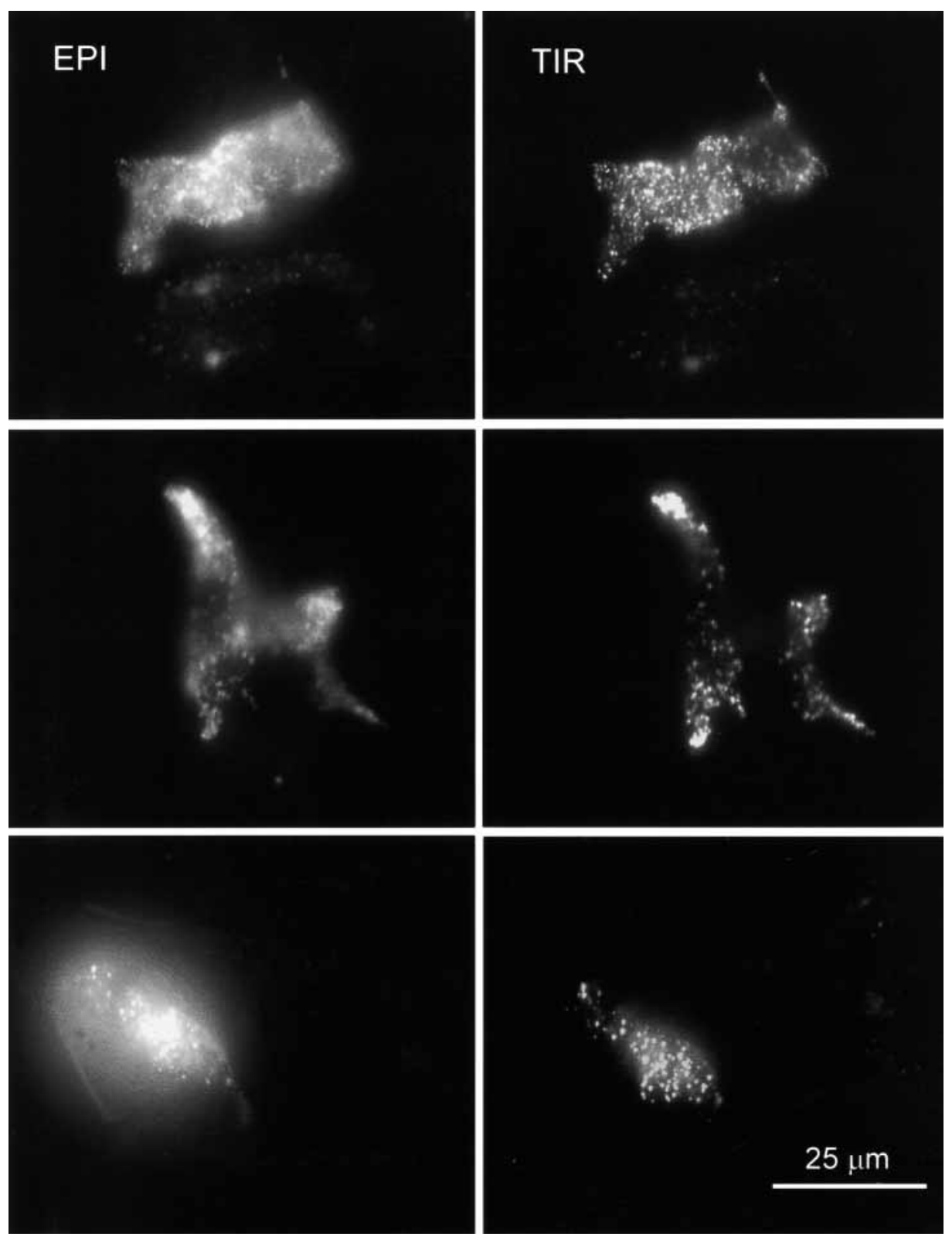

Figure 1: EPI vs. TIR prismless fluorescence photographs with the Olympus 1.45 NA $60 \mathrm{X}$ objective and an argon laser source of wavelength $488 \mathrm{~nm}$, using the side-port configuration depicted in Figure 5(A). These are three different bovine chromaffin cells containing secretory granules marked with GFP-atrial naturetic protein. The images were recorded by a cooled monochrome CCD camera (Photometrics Star-1).

tation light only at their cell/substrate contact regions but not through their bulk, they tend to survive longer under observation, thereby enabling time-lapse recording of a week in duration. During this time, newly appearing cell surface receptors can be immediately marked by fluorescent ligand that is continually present in the full cell culture medium while maintaining a low fluorescence background (45).

(g) Comparison of membrane-proximal ionic transients with simultaneous transients deeper in the cytoplasm. Since TIRF is completely compatible with standard epi-fluorescence, bright field, dark field, or phase contrast illumination, these methods of illumination can be switched back and forth rapidly by electro-optic devices (46).

\section{Theory of TIRF}

The thin layer of illumination is an 'evanescent field' which exponentially decays in intensity with increasing distance normal to the surface. The evanescent field is produced by an excitation light beam traveling in a solid (e.g. a glass coverslip or tissue culture plastic) incident at a high angle $\theta$ upon the solid/liquid surface at which the sample (e.g. single molecules or cells) adhere. That angle $\theta$, measured from the normal, must be large enough for the beam to totally internally reflect (TIR) rather than refract through the interface, a condition that occurs above some 'critical angle'. TIR generates a very thin electromagnetic field in the liquid with the same frequency as the incident light, exponentially decaying 


\section{Axelrod et al.}

in intensity with distance from the surface. This field is capable of exciting fluorophores near the surface while avoiding excitation of a possibly much larger number of fluorophores farther out in the liquid.

The simplest case of TIR is that of an 'infinitely' extended plane wave incident upon a single interface (i.e. a beam width many times the wavelength of the light, which is a good approximation for unfocused or weakly focused light). When a light beam propagating through a transparent medium 3 of high index of refraction (e.g. glass) encounters a planar interface with medium 1 of lower index of refraction (e.g. water), it undergoes total internal reflection for incidence angles (measured from the normal to the interface) greater than the 'critical angle'. The critical angle $\theta_{\mathrm{c}}$ for TIR is given by:

$$
\theta_{\mathrm{C}}=\sin ^{-1}\left(n_{1} / n_{3}\right)
$$

where $n_{1}$ and $n_{3}$ are the refractive indices of the liquid and the solid, respectively. Ratio $n_{1} / n_{3}$ must be less than unity for TIR to occur. (A refractive index $n_{2}$ will refer to an optional intermediate layer to be discussed below.) For incidence angle $\theta<\theta_{\mathrm{c}}$, most of the light propagates through the interface with a refraction angle (also measured from the normal) given by Snell's Law. (Some of the incident light also internally reflects back into the solid). For $\theta>\theta_{\mathrm{c}}$, all of the light reflects back into the solid. Some of the incident energy, however, penetrates through the interface and propagates parallel to the surface in the plane of incidence. The field in the liquid, called the 'evanescent field' (or 'wave'), is capable of exciting fluorescent molecules that might be present near the surface.

For an infinitely wide beam the intensity of the evanescent wave (measured in units of energy/area/s) exponentially decays with perpendicular distance $z$ from the interface:

$$
/(z)=/(0) e^{-z / d}
$$

where

$$
d=\frac{\lambda_{\mathrm{o}}}{4 \pi}\left(n_{3}^{2} \sin ^{2} \theta-n_{1}{ }^{2}\right)^{-1 / 2}
$$

$\lambda_{\mathrm{o}}$ is the wavelength of the incident light in vacuum. Depth $d$ is independent of the polarization of the incident light and decreases with increasing $\theta$. Except for $\theta \rightarrow \theta_{\mathrm{C}}$ (where $d \rightarrow \infty$ ), $d$ is in the order of $\lambda_{\mathrm{o}}$ or smaller.

Although the emission by a microscopic object from a fluorophore excited by the incident evanescent field might be expected to follow an exponential decay with z according to Equation 2, this is not precisely true. Fluorescence emission near a dielectric interface is rather anisotropic and the degree of anisotropicity is itself z-dependent $(47,48)$.

The polarization (i.e. the vector direction of the electric field) of the evanescent wave depends on the incident light polarization, which can be either 's' (polarized normal to the plane of incidence formed by the incident and reflected rays) or ' $p$ ' (polarized in the plane of incidence). For s-polarized incident light, the evanescent electric field vector direction remains purely normal to the plane of incidence. For p-polarized incident light, the evanescent electric field vector direction remains in the plane of incidence, but it 'cartwheels' along the surface with a nonzero longitudinal component (see Figure 2). This feature distinguishes evanescent light from freely propagating subcritical refracted light, which has no longitudinal component. The longitudinal component approaches zero as the incidence angle is reduced from the supercritical range back toward the critical angle.

A p-polarized evanescent field can be uniquely utilized to highlight submicroscopic irregularities in the plasma membrane of carbocyanine dye-labeled living cells (41), as shown schematically in Figure 3.

Intensities $I_{p, s}(0)$ are plotted vs. $\theta$ in Figure 4 . The evanescent intensity approaches zero as $\theta \rightarrow 90^{\circ}$. On the other hand, for super-critical angles within 10 degrees of $\theta_{\mathrm{C}}$, the evanescent intensity is as great as or greater than the incident light intensity. The plots can be extended without breaks to the subcritical angle range, where the intensity is that of the freely propagating refracted light in medium 1.

\section{Intermediate layers}

In actual experiments in biophysics or cell biology, the interface may not be a simple interface between two media, but rather a stratified multilayer system. One example is the case of a biological membrane or lipid bilayer interposed between glass and an aqueous medium. Another example is a thin metal film coating, which can be used to quench fluorescence within the first $\sim 10 \mathrm{~nm}$ of the surface. We discuss here the TIR evanescent wave in a three-layer system in which incident light travels from medium 3 (refractive index $\left.n_{3}\right)$ through the intermediate layer $\left(n_{2}\right)$ toward medium $1\left(n_{1}\right)$. Qualitatively, several features can be noted:

(a) Insertion of an intermediate layer never thwarts TIR, regardless of the intermediate layer's refractive index $n_{2}$. The only question is whether TIR takes place at the $n_{3}: n_{2}$ interface or the $n_{2}: n_{1}$ interface. Since the intermediate layer is likely to be very thin (no deeper than several tens of nanometers) in many applications, precisely which interface supports TIR is not important for qualitative studies.

(b) Regardless of $n_{2}$ and the thickness of the intermediate layer, the evanescent wave's profile in medium 1 will be exponentially decaying with a characteristic decay distance given by Equation 3. However, the overall distance of penetration of the field measured from the surface of medium 3 is affected by the intermediate layer.

(c) Irregularities in the intermediate layer can cause scattering of incident light which then propagates in all directions in medium 1. Experimentally, scattering appears not be a problem on samples even as inhomogeneous as biological cells. 


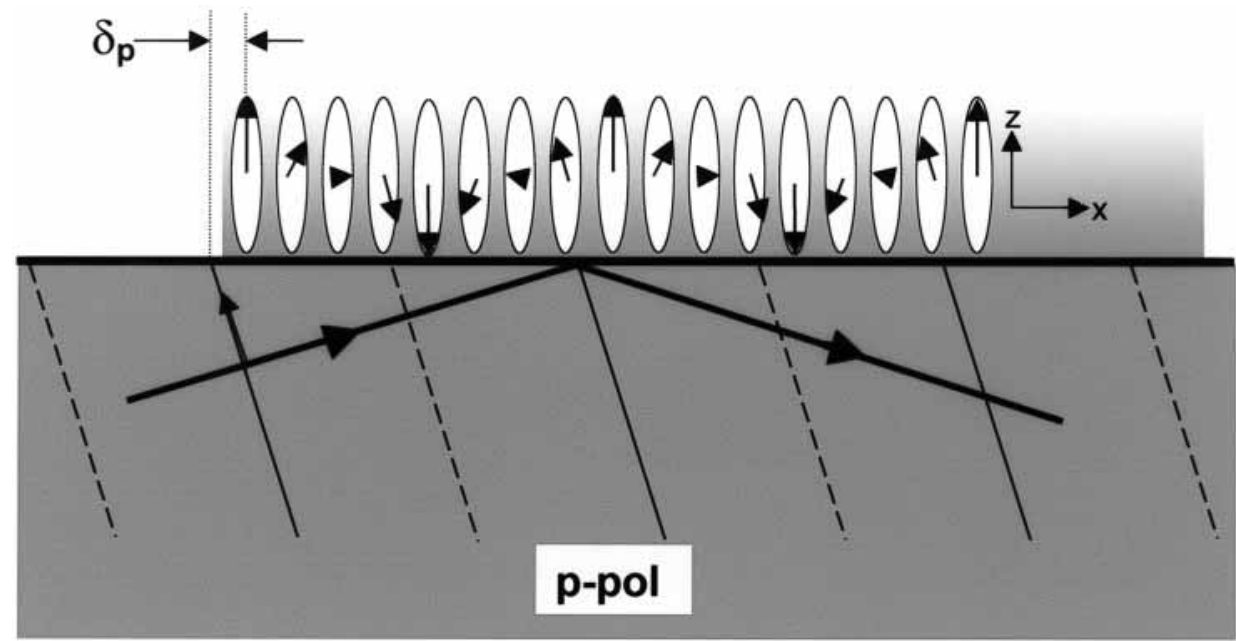

Figure 2: Schematic drawing of the evanescent polarization resulting from p-pol (in the plane of incidence) incident light. The incident light wavefronts (with the intervals from solid to dashed wavefront lines representing one-half of a wavelength in the glass) define the spacing of the spatial period along the interface, and reflected wavefronts are not shown. The p-pol evanescent field is elliptically polarized in the $x-z$ plane as shown (primarily z-pol with a weaker $x$-component at a relative phase of $\pi / 2$ ). For pictorial clarity, only two periods of evanescent electric field oscillation are shown; in reality, the evanescent region is much more extended and contains many more periods of oscillation in the $\mathrm{x}$-direction. The exact phase relationship between the incident field and the evanescent field is a function of incidence angle and is represented by $\delta_{\mathrm{p}}$ here.

Direct viewing of incident light scattered by a cell surface lying between the glass substrate and an aqueous medium confirms that scattering is many orders of magnitude dimmer than the incident or evanescent intensity, and will thereby excite a correspondingly dim contribution to the fluorescence.

A particularly interesting kind of intermediate layer is a metal film. Classical electromagnetic theory (47) shows that such a film will reduce the s-polarized evanescent intensity to nearly zero at all incidence angles. But the p-polarized behavior is quite different. At a certain sharply defined angle of incidence

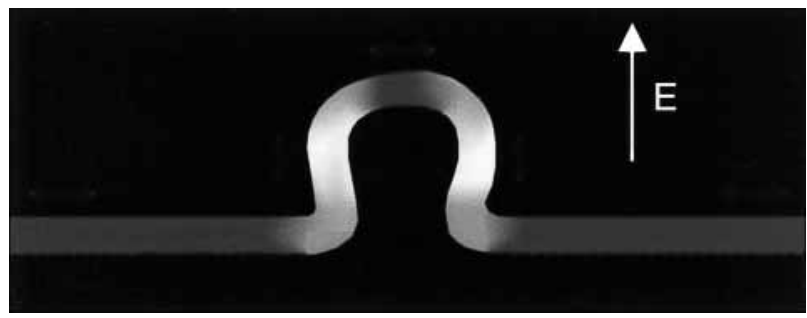

Figure 3: Schematic drawing of the excitation probability of oriented carbocyanine fluorophores embedded in a membrane in a z-polarized evanescent field (the dominant direction of a p-polarized evanescent field). The membrane is depicted in cross-section, with a curved region corresponding to a bleb or an invagination. The direction of absorption dipole of the fluorophores is known to be parallel to the local plane of the membrane and free to rotate in it (54) and is shown with bidirectional arrows. Higher excitation probability is depicted by lighter shades. The z-component of the electric field selectively excites the regions of oblique membrane orientation. $\theta_{\mathrm{p}}$ ('the surface plasmon angle'), the p-polarized evanescent intensity becomes an order of magnitude brighter than the incident light at the peak (see). This strongly peaked effect is due to a resonant excitation of electron oscillations at the metal/ water interface. For an aluminum film at a glass/water interface, $\theta_{\mathrm{p}}$ is greater than the critical angle $\theta_{\mathrm{c}}$ for TIR. The intensity

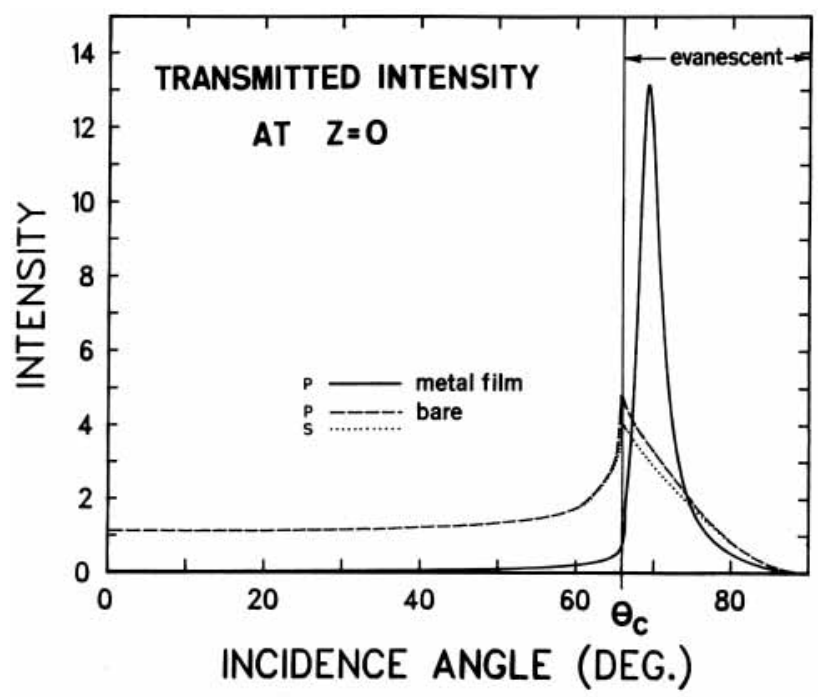

Figure 4: Evanescent intensities $I p, s$ at $z=0$ vs. $\theta$, assuming the incident intensities in the glass are set equal to unity. At angles $\theta>\theta_{\mathrm{c}}$, the transmitted light is evanescent; at angles $\theta<\theta_{\mathrm{c}}$, it is propagating. Both $s$ - and p-polarizations are shown. Refractive indices $n_{3}=1.46$ (fused silica) and $n_{1}=1.33$ are assumed here, corresponding to $\theta_{\mathrm{c}}=65.7^{\circ}$. Also shown is the evanescent intensity that would be obtained with a thin $(20 \mathrm{~nm})$ aluminum film coating 


\section{Axelrod et al.}

enhancement is rather remarkable since a $20-\mathrm{nm}$ thick metal film is almost opaque to the eye.

There are some potentially useful experimental consequences of TIR excitation through a thin metal film coated on glass:

(a) The metal film will almost totally quench fluorescence within the first $10 \mathrm{~nm}$ of the surface, and the quenching effect is virtually gone at a distance of $100 \mathrm{~nm}$. Therefore, TIR with a metal-film coated glass can be used to selectively excite fluorophores in the $10 \mathrm{~nm}$ to $200 \mathrm{~nm}$ distance range.

(b) A light beam incident upon a 20-nm aluminum film from the glass side at a glass/aluminum film/water interface evidently does not have to be collimated to produce TIR. Those rays that are incident at the surface plasmon angle will create a strong evanescent wave; those rays that are too low or high in incidence angle will create a negligible field in the water. This phenomenon may ease the practical requirement for a collimated incident beam in TIR and make it easier to set up TIR with a conventional arc light source.

(c) The metal film leads to a highly polarized evanescent wave, regardless of the purity of the incident polarization.

\section{Optical Configurations}

A wide range of optical arrangements for TIRF has been employed. Some configurations use a prism to direct the light toward the TIR interface, and others use a high numerical aperture $(N A>1.4)$ microscope objective itself for this purpose. This section gives examples of these arrangements. For concreteness in the descriptions, we assume that the sample consists of fluorescence-labeled cells in culture adhered to a glass coverslip.

\section{'Prismless' TIR through a high aperture objective}

By using an objective with a sufficiently high NA, supercritical angle incident light can be cast upon the sample by illumination through the objective $(49,50)$. The incident beam must be constrained to pass through the periphery of the objective's pupil and must emerge with only a narrow spread of angles; this can be accomplished by setting the incident beam to be focused off-axis at the objective's back focal plane. The farther the beam focus is positioned radially offaxis, the larger the angle that the beam will emerge from the objective. It can emerge into the immersion oil (refractive index $n_{\text {oil }}$ ) at a maximum possible angle $\theta_{\mathrm{m}}$ measured from the optical axis) given by:

$$
\mathrm{NA}=n_{\text {oil }} \sin \theta_{\mathrm{m}}
$$

Since $n_{i} \sin \theta_{t}$ is conserved (by Snell's Law) as the beam traverses through planar interfaces from one material (denoted with subscript i) to the next, the right side of Equation 4 is equal to $n_{3} \sin \theta_{3}$ (where subscript 3 refers to coverslip sub- strate upon which the cells grow). For total internal reflection to occur at the interface with an aqueous medium of refractive index $n_{1}, \theta_{3}$ must be greater than the critical angle $\theta_{\mathrm{c}}$ given by:

$$
n_{1}=n_{3} \sin \theta_{\mathrm{c}}
$$

From Equations 4 and 5, it is evident that the NA must be greater than $n_{1}$, preferably by a substantial margin. This is no problem for an interface with water with $n_{1}=1.33$ and a $\mathrm{NA}=1.4$ objective. But for viewing the inside of a cell at $n_{1}=$ 1.38, a NA=1.4 objective will produce TIR at only barely above the critical angle. The evanescent field in this case will be quite deep, and dense heterogeneities in the sample (such as cellular organelles) will convert some of the evanescent field into scattered propagating light.

Fortunately, objectives are now available with NA $>1.4$. The highest aperture available is an Olympus 100X NA=1.65; this works very well for through-the-lens TIR on living cells. However, that objective requires the use of expensive 1.78 refractive index coverslips made of either LAFN21 (available from Olympus) or SF11 glass (custom cut by VA Optical Co, San Anselmo, CA, USA). SF11 glass is the less expensive of the two, but it may have a chromatic dispersion not as well suited to the objective, requiring slight refocusing for different fluorophores. The 1.65 objective also requires special $n=1.78$ oil which is volatile and leaves a crystalline residue. Two other objectives that are now available circumvent these problems: an Olympus 60X NA $=1.45$ and a Zeiss $100 \times N A=1.45$. The 1.45 objectives use standard glass (1.52 refractive index) coverslips and oil and yet have an aperture adequate for TIR on cells The 1.45 objective is probably the method of choice for TIR, except for cells which have particularly dense organelles.

Configuring a microscope through-the-lens TIRF excited by a laser beam can be done either by fairly simple custombuilt or commercial accessories (Figure 5). The angle of the illumination can be continuously switched all the way from standard epi to TIR simply by increasing the off-axis position of the beam focus at the objective's back focal plane. Once TIR is achieved, further increases in off-axis position serve to increase the incidence angle at the sample and thereby decrease the depth of the evanescent field.

An arc-lamp illumination system, rather than a laser, can also be configured for TIRF illumination by use of an opaque disk of the correct diameter inserted in a plane equivalent (but upbeam) from the objective back focal plane (Figure 5D). This allows only rays at significantly off-axis radii in the back focal plane to propagate through to the TIR sample plane, upon which they are incident at supercritical angles. Switching back and forth between epi and TIR can be done simply by placing or removing the opaque disk as shown. The size of the TIR fluorescence area on the sample increases with the angle of convergence of the rays at the back focal plane. In the case of TIR illumination, this is easily increased by expanding the beam 


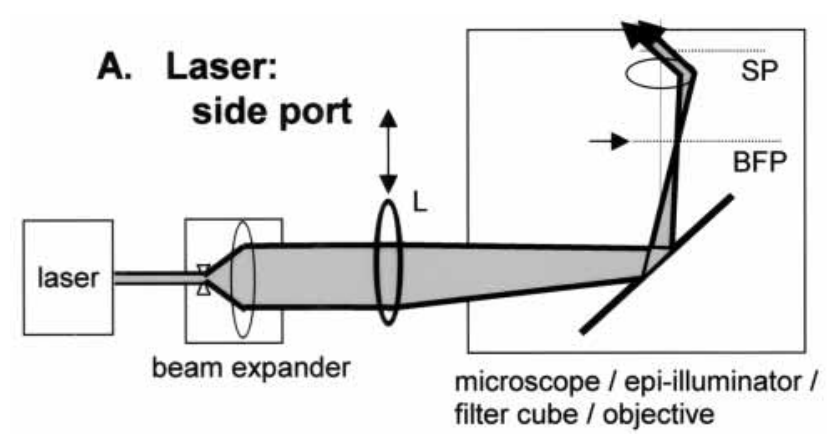

C. Laser: rear port w/ BFP

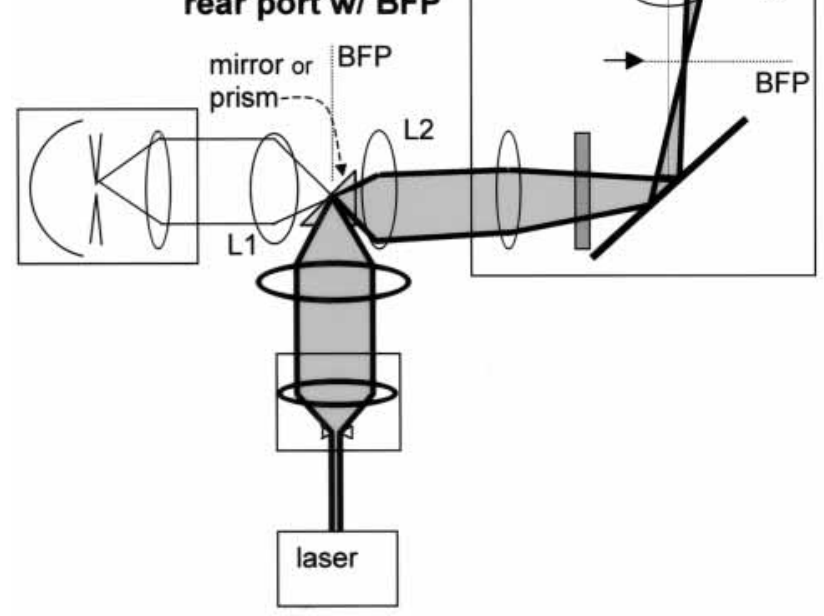

\section{B. Laser optical fiber: rear port w/o BFP}
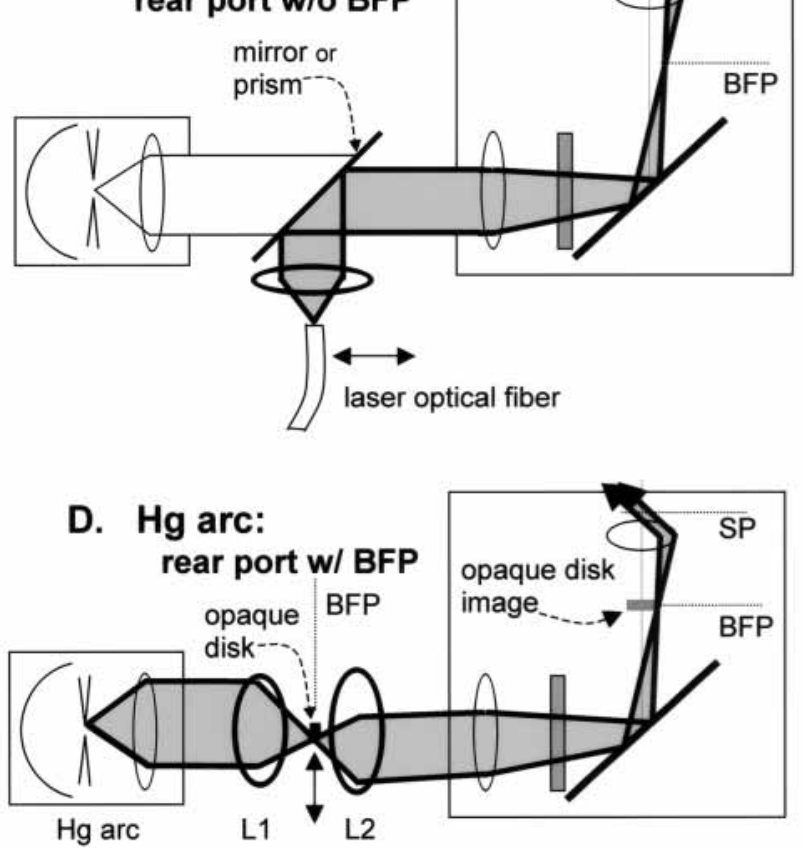

Figure 5: Four arrangements for prismless TIRF in an inverted microscope. In all these configurations, SP refers to sample plane, and BFP refers to the objective's back focal plane or its equivalent planes (also called 'aperture planes'). Components drawn with heavier lines need to be installed; components in lighter lines are possibly pre-existing in the standard microscope. (A) Laser illumination through a side port (requires a special dichroic mirror cube facing the side, available for the Olympus IX-70 microscope). The beam is focused at the back focal plane at a radial position sufficient to lead to supercritical angle propagation into the coverslip. Moving the lens $L$ transversely changes the angle of incidence at the sample and allows switching between subcritical (EPI) and supercritical (TIR) illumination. This is how Figure 1 was produced. (B) Laser illumination introduced by an optical fiber through the rear port normally used by the arc lamp, This scheme is employed by the commercial Olympus TIRF device. (C) Laser illumination in microscope systems containing an equivalent BFP in the rear path normally used by an arc lamp. The laser beam is focused at the BFP where the arc lamp would normally be imaged. The Zeiss Axiovert 200 provides this BFP, marked as an 'aperture plane'. If (as in the Olympus IX-70) an aperture plane does not exist in the indicated position, it can be created with the pair of lens L1 and L2. (D) Arc lamp TIR illumination with no laser at all. The goal is to produce a sharp-edged shadow image of an opaque circular disk at the objective back focal plane such that only supercritical light passes through the objective. The actual physical opaque disk (ideally made of aluminized coating on glass) must be positioned at an equivalent upbeam BFP which, in Kohler illumination, also contains a real image of the arc. The Zeiss Axiovert 200 provides this BFP, marked as an 'aperture plane'. If (as in the Olympus IX-70) an aperture plane does not exist in the indicated position, it can be created with the pair of lens L1 and L2. The illumination at the back focal plane is a circular annulus; it is shown as a point on one side of the optical axis for pictorial clarity only. The through-the-lens arc-lamp TIRF configuration D can be switched easily to laser TIRF configuration C by insertion of the reflecting prism in the arc lamp light path.

width at the focusing lens just before the beam enters the microscope. Arc illumination has the advantages of easy selection of excitation colors with filters and freedom from coherent light interference fringes, but it is somewhat dimmer because much of the arc lamp power directed toward the sample at subcritical angles is necessarily blocked.
The evanescent illumination is not 'pure' with through-thelens prismless TIRF: a small fraction of the illumination of the sample results from excitation light scattered within the objective, and a small fraction of the observed fluorescence arises from luminescence of the objective's internal elements. 
Axelrod et al.
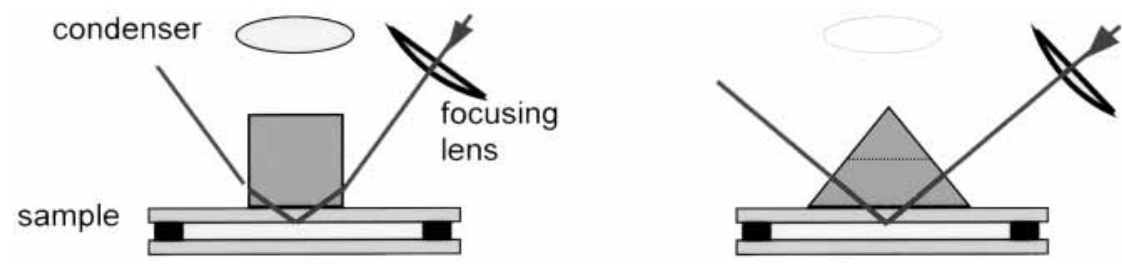

objective
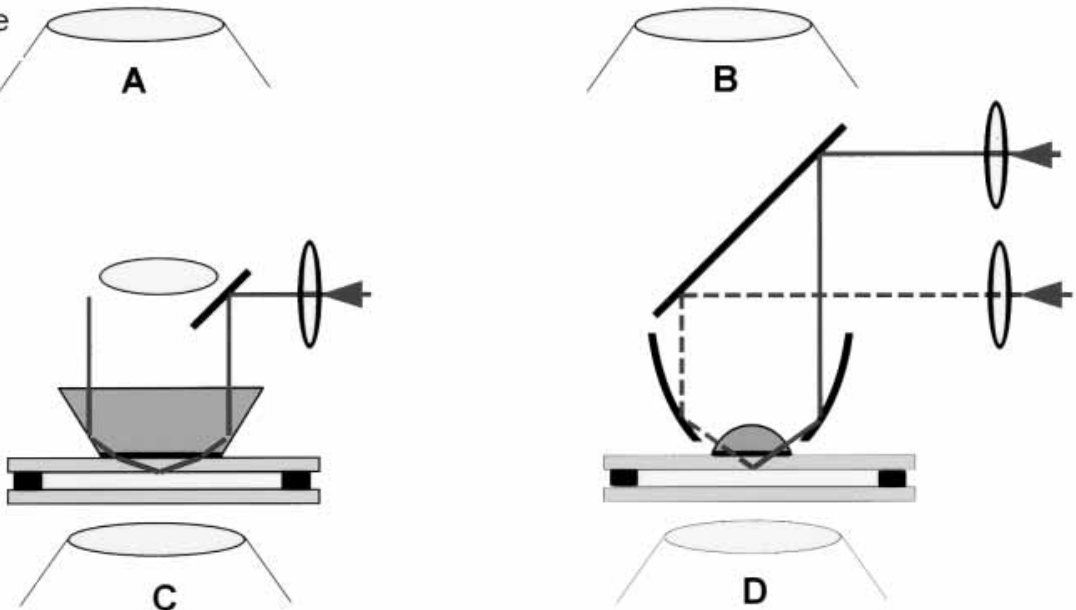

C

D

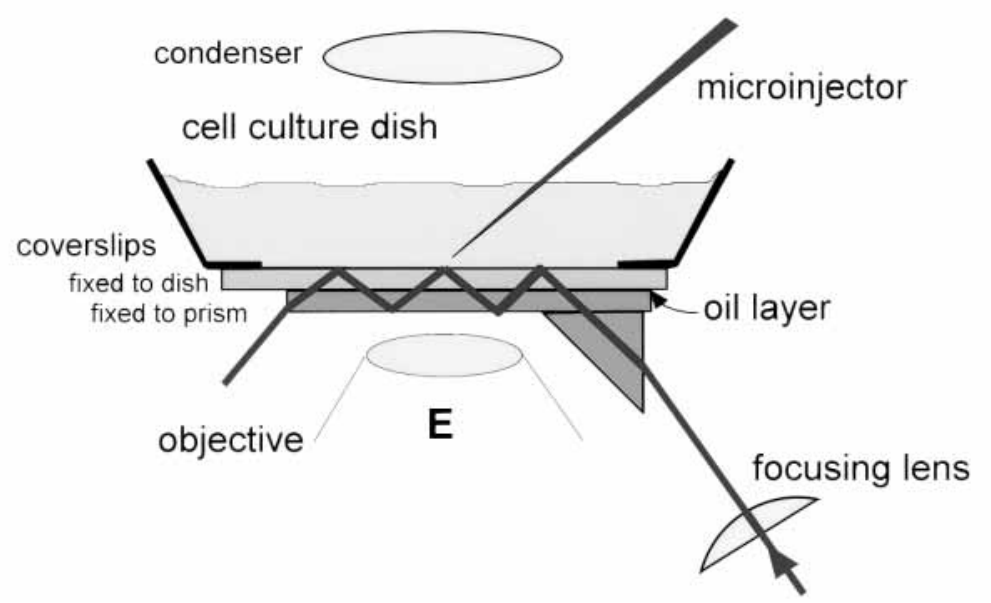

Figure 6: Schematic drawings for prism-based TIR in an inverted microscope, all using a laser as a light source. The vertical distances are exaggerated for clarity. The first four configurations (A-D) use a TIR prism above the sample. In these configurations, the buffer-filled sample chamber consists of a lower bare glass coverslip, a spacer ring (made of 60- $\mu$ m-thick Teflon or double-stick cellophane tape) and the cell coverslip inverted so the cells face down. The upper surface of the cell coverslip is put in optical contact with the prism lowered from above by a layer of immersion oil or glycerol. The lateral position of the prism is fixed but the sample can be translated while still maintaining optical contact. The lower coverslip can be oversized and the Teflon spacer can be cut with gaps so that solutions can be changed by capillary action with entrance and exit ports. Alternatively, commercially available solution-changing cell chambers (e.g. Sykes-Moore chamber from Bellco Glass Co. or rectangular cross-section microcapillary tubes from Wilmad Glass Co.) can be used. In configuration D, two incident beams split from the same laser intersect at the TIR surface, thereby setting up a striped interference pattern on the sample which is useful in studying surface diffusion (34). Configuration E places the prism below the sample and depends on multiple internal reflections in the substrate. This configuration thereby allows complete access to the sample from above for solutions changing and/or electrophysiology studies. However, only air or water immersion objectives may be used because oil at the substrate's lower surface will thwart the internal reflections.

\section{TIRF with a prism}

Although a prism may restrict sample accessibility or choice of objectives in some cases, prism-based TIR is very inexpensive to set up and produces a 'cleaner' evanescent-excited fluorescence than prismless TIR. Figure 6 shows several schematic drawings designated A-E for setting up laser/prism-based TIR in an inverted microscope. 
Figure 7 shows an exceptionally convenient (and low-cost) prism-based TIRF setup for an upright microscope. The laser beam is introduced in the same port in the microscope base as intended for the transmitted light illuminator (which should be removed), thereby utilizing the microscope's own in-base optics to direct the beam vertically upward. An extra lens just upbeam from the microscope base weakly focuses the TIR spot and permits adjustment of its position. This system gives particularly high-quality images if a water immersion objective is employed and submerged directly into the buffer solution in an uncovered cell chamber. This system is also easily used with cells adhering directly on tissue culture plastic dishes rather than on coverslips; the plastic/cell interface is then the site of TIR. If the objective has a long enough working distance, reasonable accessibility to micropipettes is possible. In this configuration, flexibility in incidence angle (to obtain a range of evanescent field depths) is sacrificed in exchange for convenience; however, a set of various-angled trapezoids will allow one to employ various discrete incidence angles. In an alternative approach for varying incidence angles over a continuous range, a hemispherical prism can be substituted for the trapezoidal prism (51). The incident laser beam is directed along a radius line at an angle set by external optical elements.

In all these prism-based methods, choice of optical materials is somewhat flexible, as follows. (a) The prism used to couple the light into the system and the (usually disposable) slide or coverslip in which TIR takes place need not be matched exactly in refractive index. (b) The prism and slide may be optically coupled with glycerol, cyclohexanol, or microscope immersion oil, among other liquids. Immersion oil has a higher refractive index (thereby avoiding possible TIR at the prism/coupling liquid interface at low incidence angles) but it tends to be more autofluorescent (even the 'extremely low' fluorescence types). (c) The prism and slide can both be made of ordinary optical glass for many applications, unless shorter penetration depths arising from higher refractive indices are desired. Optical glass does not transmit light below about $310 \mathrm{~nm}$ and also has a dim autoluminescence with a long (several hundred microsecond) decay time, which can be a problem in some fluorescence recovery after photobleaching (FRAP) experiments. The autoluminescence of high-quality fused silica (often called 'quartz') is much lower. Tissue culture dish plastic (particularly convenient as a substrate in the upright microscope setup) is also suitable, but tends to have a significant autofluorescence compared to ordinary glass. More exotic high $n_{3}$ materials such as sapphire, titanium dioxide, and strontium titanate can yield exponential decay depths $d$ as low as $\lambda_{0} / 20$.

In all the prism-based methods, the TIRF spot should be focused to a width no larger than the field of view; the larger the spot, the more that spurious scattering and out-of-focus fluorescence from the immersion oil layer between the prism and coverslip will increase the generally very low fluorescence background attainable by TIRF. Also, the incidence angle should exceed the critical angle by at least a couple of degrees. At incidence angles very near the critical angle, the cells cast a noticeable 'shadow' along the surface.

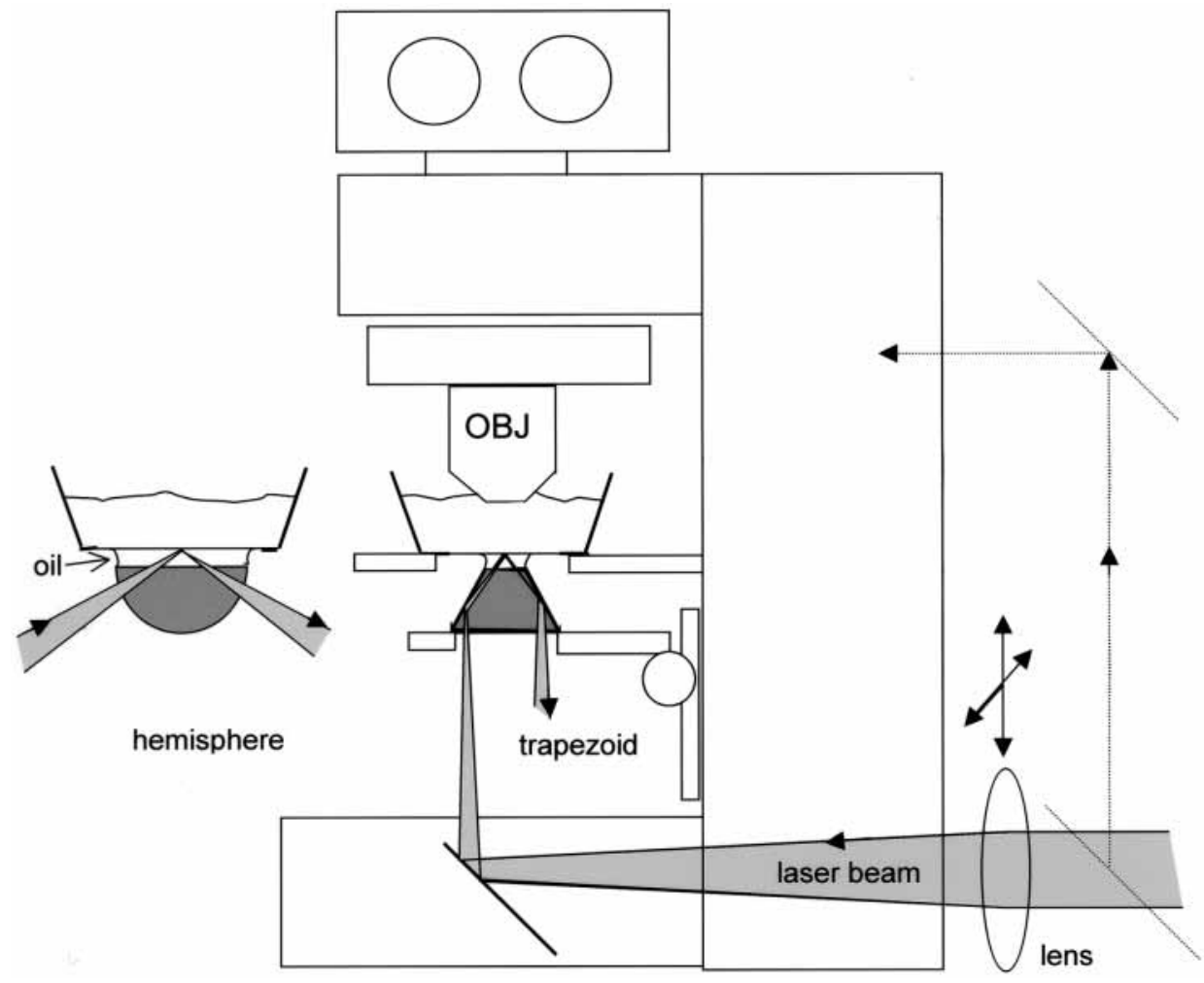

Figure 7: TIRF for an upright microscope utilizing the integral optics in the microscope base and a trapezoidal prism on the condenser mount and movable up and down. The position of the beam is adjustable by moving the external lens. An alternative hemispherical prism configuration for variable incidence angle is also indicated to the left. Vertical distances are exaggerated for clarity. An extra set of mirrors can be installed to deflect the beam into an epi-illumination light path (shown with dashed lines). 
Axelrod et al.

\section{General Experimental Considerations}

A step-by-step guide for setting up TIRF in a standard fluorescence microscope has been published (52).

\section{Laser source}

A laser with a total visible output in the $100 \mathrm{~mW}$ or greater range should be adequate for most TIRF applications. Aircooled argon or diode lasers of less than $100 \mathrm{~mW}$ are adequate for some purposes, but probably marginal for dim samples or for samples where a weaker laser line (e.g. the $457 \mathrm{~nm}$ line of argon) may be desired to excite a shorter wavelength fluorescent marker (such as cyan fluorescent protein CFP). Laser illumination produces interference fringes which are manifested as intensity variations over the sample area. For critical applications, it may be advisable to rapidly jiggle the beam (e.g. in a commercially available optical fiber mode scrambler) or to compute a normalization of sample digital images against a control digital image of a uniform concentration of fluorophores.

\section{Functionalized substrates}

TIRF experiments often involve specially coated substrates. A glass surface can be chemically derivatized to yield special physi- or chemi-absorptive properties. Covalent attachment of certain specific chemicals are particularly useful in cell biology and biophysics, including: poly I-lysine for enhanced adherence of cells; hydrocarbon chains for hydrophobicizing the surface in preparation for lipid monolayer adsorption; and antibodies, antigens, or lectins for producing specific reactivities. Derivatization generally involves pretreatment of the glass by an organosilane.

A planar phospholipid coating (possibly with incorporated proteins) on glass can be used as a model of a biological membrane. Methods for preparing such model membranes on planar surfaces suitable for TIR are reviewed by Thompson et al. (53).

Aluminum coating (for surface fluorescence quenching) can be accomplished in a standard vacuum evaporator; the amount of deposition can be made reproducible by completely evaporating a premeasured constant amount of aluminum. After deposition, the upper surface of the aluminum film spontaneously oxidizes in air very rapidly. This aluminum oxide layer appears to have some similar chemical properties to the silicon dioxide of a glass surface; it can be derivatized by organosilanes in much the same manner.

\section{Photochemistry at the surface}

Illumination of surface-adsorbed proteins can lead to apparent photochemically induced crosslinking. This effect is observed as a slow, continual, illumination-dependent increase in the observed fluorescence. It can be inhibited by deoxygenation (aided by the use of an $\mathrm{O}_{2}$-consuming enzyme/substrate system such as protocatachuic deoxygenase/protocatachuic acid or a glucose/glucose oxidase system), or by $0.05 \mathrm{~m}$ cysteamine.

\section{TIRF vs. Other Optical Section Microscopies}

Confocal microscopy (CM) is another technique for apparent optical sectioning, achieved by exclusion of out-of-focus emitted light with a set of image plane pinholes. CM has the clear advantage in versatility; its method of optical sectioning works at any plane of the sample, not just at an interface between dissimilar refractive indices. However, other differences exist which, in some special applications, can favor the use of TIRF: (a) The depth of the optical section in TIRF is typically $\leq 0.1 \mu \mathrm{m}$, whereas in $\mathrm{CM}$ it is a relatively thick $\sim 0.6 \mu \mathrm{m}$. (b) In some applications (e.g. FRAP, FCS, or on cells whose viability is damaged by light), illumination and not just detected emission is best restricted to a thin section; this is possible only with TIRF. (c) Since TIRF can be adapted to and made interchangeable with existing standard microscope optics, even with 'home-made' components, it is much less expensive than CM. Laser-based TIRF microscopy kits are also now available commercially from Olympus and from Till Photonics.

Two-photon microscopy (TPM) (55) has many desirable features, including true optical sectioning, whereby the plane of interest is the only one that is actually excited, as in TIRF. TPM is not restricted to the proximity of an interface, but the optical section of TPM is still much thicker than that of TIRF.

Cell-substrate contacts can be located by a nonfluorescence technique completely distinct from TIRF, known as 'internal reflection microscopy' (IRM). Using conventional illumination sources, IRM visualizes cell-substrate contacts as dark regions. IRM has the advantage that it does not require the cells to be labeled, but the disadvantages that it contains no information on biochemical specificities in the contact regions and that it is less sensitive to changes in contact distance (relative to TIRF) within the critical first $100 \mathrm{~nm}$ of the surface.

\section{Acknowledgments}

This work was supported by NIH grant 5 R01 NS38129. The author wishes to thank Drs Ron Holz and Edwin Levitan for the chromaffin cells and the GFP construct, respectively, depicted in Figure 1. The author also thanks all the past and present graduate students and postdocs who have contributed to aspects of the TIRF work described here: Thomas Burghardt, Nancy Thompson, Edward Hellen, Andrea Stout, Ariane Mc Kiernan, Michelle Dong Wang, Robert Fulbright, Laura Johns, Susan Sund, and Miriam Allersma.

\section{References}

1. Axelrod D. Cell surface contacts illuminated by total internal reflection fluorescence. J Cell Biol 1981;89:141-145.

2. Weis RM, Balakrishnan K, Smith B, McConnell HM. Stimulation of fluorescence in a small contact region between rat basophil leukemia cells and planar lipid membrane targets by coherent evanescent radiation. J Biol Chem 1982;257:6440-6445. 
3. Gingell D, Heavens OS, Mellor JS. General electromagnetic theory of internal reflection fluorescence: the quantitative basis for mapping cell-substratum topography. J Cell Sci 1987:87:677-693.

4. Todd I, Mellor JS, Gingell D. Mapping of cell-glass contacts of Dictyostelium amoebae by total internal reflection aqueous fluorescence overcomes a basic ambiguity of interference reflection microscopy. J Cell Sci 1988;89:107-114.

5. Lang T, Wacker I, Wunderlich I, Rohrbach A, Giese G, Soldati T, Almers $\mathrm{W}$. Role of actin cortex in the subplasmalemmal transport of secretory granules in PC-12 cells. Biophys J 2000;78:2863-2877.

6. Vale RD, Funatsu T, Pierce DW, Romberg L, Harada Y, Yanagida T. Direct observation of single kinesin molecules moving along microtubules. Nature 1996:380:451-453.

7. Khan S, Pierce D, Vale RD. Interactions of the chemotaxis signal protein CheY with bacterial flagellar motors visualized by evanescent wave microscopy. Curr Biol 2000;10:927-930.

8. Dickson RM, Norris DJ, Tzeng Y-L, Moerner WE. Three-dimensional imaging of single molecules solvated in pores of poly (acrylamide) gels. Science 1996;274:966-969.

9. Dickson RM, Norris DJ, Moerner WE. Simultaneous imaging of individual molecules aligned both parallel and perpendicular to the optic axis. Phys Rev Lett 1998;81:5322-5325.

10. Sako Y, Miniguchi S, Yanagida T. Single-molecule imaging of EGFR signalling on the surface of living cells. Nature Cell Biol 2000;2:168-172.

11. Knight AE, Molloy JE. Muscle, myosin and single molecules. In: Higgins, SJ Banting, G, eds. Molecular Motors. Essays in Biochemistry, Vol. 35. London: Portland Press; 2000. p. 200

12. Ha TJ, Ting AY, Liang J, Caldwell WB, Deniz AA, Chemla DS, Schultz PG, Weiss S. Single-molecule fluorescence spectroscopy of enzyme conformational dynamics and cleavage mechanism. Proc Natl Acad Sci USA 1999;96:893-898.

13. Starr TE, Thompson NL. Total internal reflection with fluorescence correlation spectroscopy: combined surface reaction and solution diffusion. Biophys J 2001:80:1575-1584.

14. Lang T, Wacker I, Steyer J, Kaether C, Wunderlich I, Soldati T, Gerdes $\mathrm{H}-\mathrm{H}$, Almers $\mathrm{W} . \mathrm{Ca}^{2+}$.triggered peptide secretion neurotechnique in single cells imaged with green fluorescent protein and evanescentwave microscopy. Neuron 1997;18:857-963.

15. Steyer JA, Almers W. Tracking single secretory granules in live chromaffin cells by evanescent-field fluorescence microscopy. Biophys J 1999:76:2262-2271.

16. Toomre D, Steyer JA, Keller P, Almers W, Simons K. Fusion of constitutive membrane traffic with the cell surface observed by evanescent wave microscopy. J Cell Biol 2000;149:33-40.

17. Zenisek DP, Steyer JA, Almers W. Imaging exocytosis of single synaptic vesicles with evanescent field microscopy. Biophys J 2000;78: 1538.

18. Steyer JA, Almers W. A real-time view of life within $100 \mathrm{~nm}$ of the plasma membrane. Nature Rev Mol Cell Biol $2001 ; 2: 268-275$.

19. Lang T, Bruns D, Wenzel D, Riedel D, Holroyd P, Thiele C, Jahn R. SNAREs are concentrated in cholesterol-dependent clusters that define docking and fusion sites for exocytosis. EMBO J 2001;20:22022213.

20. Oheim M, Loerke D, Stuhmer W, Chow RH. The last few milliseconds in the life of a secretory granule. Docking, dynamics and fusion visualized by total internal reflection fluorescence microscopy (TIRFM). Eur Biophys J 1998;27:83-98.

21. Oheim M, Loerke D, Stuhmer W, Chow RH. Multiple stimulation-dependent processes regulate the size of the releasable pool of vesicles. Eur Biophys J 1999:28:91-101.

22. Oheim M, Stuhmer $W$. Interaction of secretory organelles with the membrane. J Memb Biol 2000;178:163-173.

23. Han W, Ng Y-K, Axelrod D, Levitan ES. Neuronal peptide release is sustained by recruitment of rapidly diffusing secretory vesicles. Proc Natl Acad Sci USA 1999;96:14577-14582.
24. Schmoranzer J, Goulian M, Axelrod D, Simon SM. Imaging constitutive exocytosis with total internal reflection microscopy. J Cell Biol 2000;149:23-31.

25. Johns LM, Levitan ES, Shelden EA, Holz RW, Axelrod D. Restriction of secretory granule motion near the plasma membrane of chromaffin cells. J Cell Biol 2001;153:177-190.

26. Tsuboi T, Zhao C, Terakawa S, Rutter GA. Simultaneous evanescent wave imaging of insulin vesicle membrane and cargo during a single exocytotic event. Curr Biol 2000;10:1307-1310.

27. Tsuboi T, Kikuta T, Warashina A, Terakawa S. Protein kinase C-dependent supply of secretory granules to the plasma membrane. Biochem Biophys Res Comm 2001;282:621-628.

28. Rohrbach A. Observing secretory granules with a multiangle evanescent wave microscope. Biophys J 2000;78:2641-2654.

29. Toomre D, Manstein DJ. Lighting up the cell surface with evanescent wave microscopy. Trends Cell Biol 2001:11:298-303.

30. Thompson NL, Burghardt TP, Axelrod D. Measuring surface dynamics of biomolecules by total internal reflection with photobleaching recovery or correlation spectroscopy. Biophys J 1981;33:435-454.

31. Burghardt TP, Axelrod D. Total internal reflection/fluorescence photobleaching recovery study of serum albumin adsorption dynamics. Biophys J 1981;33:455-468.

32. Thompson NL, Axelrod D. Immunoglobulin surface binding kinetics studied by total internal reflection with fluorescence correlation spectroscopy. Biophys J 1983:43:103-114.

33. Hellen E, Axelrod D. Kinetics of epidermal growth factor/receptor binding on cells measured by total internal reflection/fluorescence recovery after photobleaching. J Fluor 1991;1:113-128.

34. Fulbright RM, D. Axelrod. Dynamics of nonspecific adsorption of insulin to erythrocyte membrane. J Fluor 1993:3:1-16.

35. Stout AL, Axelrod D. Reversible binding kinetics of a cytoskeletal protein at the erythrocyte submembrane. Biophys J 1994;67:1324-1334.

36. McKiernan AM, MacDonald RC, MacDonald RI, Axelrod D. Cytoskeletal protein binding kinetics at planar phospholipid membranes. Biophys J 1997;73:1987-1998.

37. Sund $\mathrm{SE}$, Axelrod D. Actin dynamics at the living cell submembrane imaged by total internal reflection fluorescence photobleaching. Biophys J 2000;79:1655-1669

38. Kalb E, Engel J, Tamm LK. Binding of proteins to specific target sites in membranes measured by total internal reflection fluorescence microscopy. Biochemistry 1990;29:1607-1613.

39. Gilmanshin R, Creutz CE, Tamm LK. Annexin IV reduces the rate of lateral lipid diffusion and changes the fluid phase structure of the lipid bilayer when it binds to negatively charged membranes in the presence of calcium. Biochemistry 1994;33:8225-8832.

40. Hinterdorfer P, Baber G, Tamm LK. Reconstitution of membrane fusion sites. A total internal reflection fluorescence microscopy study of influenza hemagglutinin-mediated membrane fusion. J Biol Chem 1994:269:20360-20368.

41. Lagerholm BC, Starr TE, Volovyk ZN, Thompson NL. Rebinding of IgE Fabs at haptenated planar membranes: measurement by total internal reflection with fluorescence photobleaching recovery. Biochemistry 2000:39:2042-2051.

42. Tilton RD, Gast AP, Robertson CR. Surface diffusion of interacting proteins. Effect of concentration on the lateral mobility of adsorbed bovine serum albumin. Biophys J 1990;58:1321-1326.

43. Sund SE, Swanson JA, Axelrod D. Cell membrane orientation visualized by polarized total internal reflection fluorescence. Biophys $\mathrm{J}$ 1999;77:2266-2283.

44. Mathur AB, Truskey GA, Reichert WM. Atomic force and total internal reflection fluorescence microscopy for the study of force transmission in endothelial cells. Biophys J 2000;78:1725-1735.

45. Wang MD, Axelrod D. Time-lapse total internal reflection fluorescence video of acetylcholine receptor cluster formation on myotubes. Dev Dyn 1994:201:29-40. 
Axelrod et al.

46. Omann GM, Axelrod D. Membrane proximal calcium transients in stimulated neutrophils seen by total internal reflection fluorescence. Biophys J 1996;71:2885-2891.

47. Hellen EH, Axelrod D. Fluorescence emission at dielectric and metalfilm interfaces. J Opt Soc Am B 1987;4:337-350

48. Mertz J. Radiative absorption, fluorescence, and scattering of a classical dipole near a lossless interface: a unified description. J Opt Soc Am B 2000;17:1906-1913.

49. Stout AL, Axelrod D. Evanescent field excitation of fluorescence by epi-illumination microscopy. Appl Opt 1989;28:5237-5242.

50. Axelrod D. Surface-selective fluorescence imaging with a very high aperture objective. J Biomed Opt $2001 ; 6: 6-13$.

51. Loerke D, Preitz B, Stuhmer W, Oheim M. Super-resolution measurements with evanescent-wave fluorescence excitation using variable beam incidence. J Biomed Opt 2000;5:23-30.
52. Axelrod D. Surface fluorescence microscopy by evanescent wave illumination. In: Lacey AJ, ed. Light Microscopy in Biology - A Practical Approach, 2nd edn. Oxford: Oxford University Press; 1999. p. 390413

53. Thompson NL, Pearce KH, Hsieh HV. Total internal reflection fluorescence microscopy - application to substrate-supported planar membranes. Eur Biophys J 1993:22:367-378.

54. Axelrod D. Carbocyanine dye orientation in red cell membrane studied by microscopic fluorescence polarization. Biophys J 1979;26:557-574.

55. White JG, Squirrell JM, Eliceiri KW. Applying multiphoton imaging to the study of membrane dynamics in living cells. Traffic 2000;2:775780 . 